\title{
PRIME NUMBERS IN INTERVALS STARTING AT A FIXED POWER OF THE INTEGERS
}

\author{
DANILO BAZZANELLA
}

(Received 27 March 2007; accepted 12 November 2008)

Communicated by I. E. Shparlinski

\begin{abstract}
The best known results about the distribution of prime numbers in short intervals imply that all intervals $[n, n+H] \subset[N, 2 N]$ contain the expected number of primes for all $H \geq N^{7 / 12}$, and almost all intervals $[n, n+H] \subset[N, 2 N]$ contain the expected number of primes for all $H \geq N^{1 / 6}$. As a natural generalization, this paper is concerned with the distribution of prime numbers in intervals of type $\left[n^{\alpha}, n^{\alpha}+H\right]$ with $\alpha>1$.
\end{abstract}

2000 Mathematics subject classification: primary 11NO5.

Keywords and phrases: prime numbers in short intervals.

\section{Introduction}

Let $\psi(x)=\sum_{n \leq x} \Lambda(n)$, where $\Lambda(n)$ is the von Mangoldt function. We consider the asymptotic formula

$$
\psi(x+H)-\psi(x) \sim H \quad \text { as } x \rightarrow \infty,
$$

which is related to the number of primes in the interval $(x, x+H]$. The prime number theorem implies that (1) holds with $H \gg x$. An interval $(x, x+H]$ with $H=o(x)$ is called a short interval. The best known unconditional result about the distribution of primes in short intervals is due to Huxley [8] and asserts that (1) holds for all $H \geq x^{7 / 12+\varepsilon}$. This was slightly improved by Heath-Brown in [7] to $H \geq x^{7 / 12-o(1)}$. Under the assumption of the Riemann hypothesis, Selberg [11] proved that (1) holds for all $H \geq x^{1 / 2} f(x) \log x$ where $f(x) \rightarrow \infty$ arbitrarily slowly. These results imply that all intervals $[n, n+H] \subset[N, 2 N]$ contain the expected number of primes for all $H \geq N^{7 / 12}$ and, assuming the Riemann hypothesis, for all $H \geq N^{1 / 2} f(N) \log N$ where $f(N) \rightarrow \infty$ arbitrarily slowly.

(C) 2009 Australian Mathematical Publishing Association Inc. 1446-7887/2009 \$16.00 
We can relax our conditions and investigate if (1) holds for 'almost all' $x$. By this, we mean that the measure of $x \in[X, 2 X]$ for which (1) does not hold is $o(X)$. Huxley's zero density estimate [8], in conjunction with the method of Selberg [11], shows that (1) holds for almost all $x$ with $H \geq x^{1 / 6+\varepsilon}$, slightly improved by Zaccagnini in [14] to $H \geq x^{1 / 6-o(1)}$. Under the assumption of the Riemann hypothesis, Selberg [11] proved that (1) holds for almost all $x$ with $H \geq f(x) \log ^{2} x$, where $f(x) \rightarrow \infty$ arbitrarily slowly. These results imply that almost all intervals $[n, n+H] \subset[N, 2 N]$ contain the expected number of primes for all $H \geq N^{1 / 6}$ and, assuming the Riemann hypothesis, for all $H \geq f(N) \log ^{2} N$ with $f(N) \rightarrow \infty$ arbitrarily slowly.

As a natural generalization of the above results, this paper is concerned with the distribution of prime numbers in intervals $\left[n^{\alpha}, n^{\alpha}+H\right]$, with fixed $\alpha>1$. Our main unconditional result is as follows.

Theorem 1. Let $\varepsilon>0$ and $\alpha>1$. Then almost all intervals $\left[n^{\alpha}, n^{\alpha}+H\right] \subset$ $[N, 2 N]$ contain the expected number of primes for all $H \geq N^{c(\alpha)+\varepsilon}$, where

$$
c(\alpha)= \begin{cases}\frac{1}{6} & \text { if } 1<\alpha \leq \frac{6}{5}, \\ \frac{11 \alpha-10}{16 \alpha} & \text { if } \frac{6}{5}<\alpha \leq \frac{6}{5}+\Delta, \\ 1-\sup _{(k, l)} \frac{5(1+\alpha-l+k)}{\alpha(5 k+12)} & \text { if } \alpha \geq 4,\end{cases}
$$

with $\Delta$ suitable positive constant and $(k, l)$ running over the exponent pairs.

For the sake of simplicity, we will explicitly work out the value of the function $c(\alpha)$ only for the extreme and more interesting values of $\alpha$. However, it will be clear from the proof that the same method enables one to obtain the explicit values of the function $c(\alpha)$ in the whole range $\alpha>1$. As one might expect, we get an increasing function $c(\alpha)$ such that $c(1)=1 / 6, c(\alpha)<7 / 12$ for every $\alpha$ and

$$
\lim _{\alpha \rightarrow+\infty} c(\alpha)=\frac{7}{12} \text {. }
$$

To bound some sums which arise in our argument we employ the counting functions $N(\sigma, T)$ and $N^{*}(\sigma, T)$. The former is defined as the number of zeros $\rho=\beta+i \gamma$ of Riemann zeta function which satisfy $\sigma \leq \beta \leq 1$ and $|\gamma| \leq T$, while $N^{*}(\sigma, T)$ is defined as the number of ordered sets of zeros $\rho_{j}=\beta_{j}+i \gamma_{j}(1 \leq j \leq 4)$, each counted by $N(\sigma, T)$, for which $\left|\gamma_{1}+\gamma_{2}-\gamma_{3}-\gamma_{4}\right| \leq 1$. If we make the heuristic assumption that

$$
N^{*}(\sigma, T) \ll \frac{N(\sigma, T)^{4}}{T},
$$

as in Bazzanella and Perelli [2], then we can simplify and improve Theorem 1 for large values of $\alpha$ as follows. 
THEOREM 2. Assume (2), suppose that $\varepsilon>0$ and $\alpha \geq 4$. Then almost all intervals $\left[n^{\alpha}, n^{\alpha}+H\right] \subset[N, 2 N]$ contain the expected number of primes for all $H \geq N^{c(\alpha)+\varepsilon}$ and

$$
c(\alpha)=\frac{7}{12}-\frac{5}{12 \alpha} .
$$

We conclude by presenting our results under the assumption of more standard hypotheses.

THEOREM 3. Let $\alpha>1, \varepsilon>0$ and assume the Lindelöf hypothesis. Then almost all intervals $\left[n^{\alpha}, n^{\alpha}+H\right] \subset[N, 2 N]$ contain the expected number of primes for all $H \geq N^{c(\alpha)+\varepsilon}$ and

$$
c(\alpha)=\frac{1}{2}\left(1-\frac{1}{\alpha}\right) .
$$

THEOREM 4. Let $\alpha>1$ and assume the Riemann hypothesis. Then almost all intervals $\left[n^{\alpha}, n^{\alpha}+H\right] \subset[N, 2 N]$ contain the expected number of primes for all $H \geq N^{c(\alpha)} f(N) \log ^{2} N$ with $f(N) \rightarrow \infty$ arbitrarily slowly and

$$
c(\alpha)=\frac{1}{2}\left(1-\frac{1}{\alpha}\right) .
$$

As one might expect, under the assumption of the Lindelöf hypothesis or the Riemann hypothesis, we get an increasing function $c(\alpha)$ such that $c(1)=0, c(\alpha)<$ $1 / 2$ for every $\alpha$ and

$$
\lim _{\alpha \rightarrow+\infty} c(\alpha)=\frac{1}{2}
$$

The main tools of the proofs are the Kusmin-Landau estimate for an exponential sum together with the van der Corput's method of exponent pairs, see [4], and a result about the structure of the exceptional set for the distribution of primes in short intervals due to Bazzanella and Perelli, see [2] and [1].

\section{Definitions and basic lemmas}

Our starting point is the definition of the exceptional set for the number of primes in short intervals. Let $|\cdot|$ denote the modulus of a complex number or the Lebesgue measure of an infinite set of real numbers or the cardinality of a finite set. Let $X$ be a large positive number, $\delta>0$ and define

$$
E_{\delta}(X, H)=\{X \leq x \leq 2 X:|\psi(x+H(x))-\psi(x)-H(x)| \geq \delta H(x)\} .
$$

It is clear that (1) holds if and only if for every $\delta>0$ there exists $X_{0}(\delta)$ such that $E_{\delta}(X, H)=\emptyset$ for all $X \geq X_{0}(\delta)$. Hence for small $\delta>0, X$ tending to $\infty$, the set $E_{\delta}(X, H)$ contains the exceptions, if any, to the expected asymptotic formula for the number of primes in short intervals. We will consider increasing functions $H(x)$ of 
the form $H(x)=x^{\theta+\varepsilon(x)}$, with some $0<\theta<1$ and a differentiable function $\varepsilon(x)$ such that $|\varepsilon(x)|$ is decreasing, $\varepsilon(x)=o(1)$ and

$$
\varepsilon(x+y)=\varepsilon(x)+O\left(\frac{|y|}{x \log x}\right) .
$$

A function satisfying these requirements will be called of type $\theta$. It is easy to see that functions like $x^{\theta} \log ^{c} x$, with $c$ a real constant, and similar functions, are of type $\theta$, and that for every function $H(x)$ of type $\theta$ we have $H(2 x) \ll H(x)$.

REMARK. In a preceding paper, the author and Perelli [2] defined in a slightly different way the set of functions $H(x)$ of type $\theta$, and set

$$
\varepsilon(x+y)=\varepsilon(x)+O\left(\frac{|y|}{x}\right)
$$

instead of (3). We remark that with this weaker condition we do not have $H(2 x)$ $\ll H(x)$ as claimed.

Our first lemma is concerned with the structure of the exceptional set above.

LEMMA 1. Let $0<\theta<1$, let $H(x)$ be of type $\theta$, let $X$ be sufficiently large depending on the function $H(x)$ and let $0<\delta^{\prime}<\delta$ with $\delta-\delta^{\prime} \geq \exp (-\sqrt{\log X})$. If $x_{0} \in E_{\delta}(X, H)$ then $E_{\delta^{\prime}}(X, H)$ contains the interval $\left[x_{0}-c H(X), x_{0}+c H(X)\right] \cap$ $[X, 2 X]$, where $c=\left(\delta-\delta^{\prime}\right) \theta / 5$.

PROOF. We will always assume that $x$ and $X$ are sufficiently large as prescribed by the various statements, and $\varepsilon>0$ is arbitrarily small and not necessarily the same at each occurrence.

We first observe from the definition of a function of type $\theta$ that if $y=O\left(x^{\alpha+\varepsilon}\right)$ with some $0<\alpha<1$, then

$$
H(x+y)=H(x)+O\left(x^{\theta+\alpha-1+\varepsilon}\right)
$$

for every $\varepsilon>0$.

From the Brun-Titchmarsh theorem (see Montgomery and Vaughan [10]), we have that

$$
\psi(x+y)-\psi(x) \leq \frac{21}{10} y \frac{\log x}{\log y}
$$

for all $10 \leq y \leq x$. From (5) we easily obtain that

$$
\psi(x+y)-\psi(x) \leq \frac{9}{4 \alpha} c Y
$$

for all $X \leq x \leq 3 X$ and $0 \leq y \leq c Y$, where $0<\alpha<1, X^{\alpha-\varepsilon} \leq Y \leq X$ and

$$
\frac{\alpha}{5} \exp (-\sqrt{\log X}) \leq c \leq 1
$$


Let $H(x)$ be of type $\theta, x_{0} \in E_{\delta}(X, H)$,

$$
x \in\left[x_{0}-c H(X), x_{0}+c H(X)\right] \cap[X, 2 X],
$$

where $c$ satisfies the above restrictions, and

$$
\Delta(x, H)=\psi(x+H(x))-\psi(x)-H(x) .
$$

We have

$$
\begin{aligned}
|\Delta(x, H)|= & \left|\Delta\left(x_{0}, H\right)+\Delta(x, H)-\Delta\left(x_{0}, H\right)\right| \\
\geq & \left|\Delta\left(x_{0}, H\right)\right|-\left|\psi(x+H(x))-\psi\left(x_{0}+H\left(x_{0}\right)\right)\right| \\
& -\left|\psi(x)-\psi\left(x_{0}\right)\right|-\left|H(x)-H\left(x_{0}\right)\right| .
\end{aligned}
$$

But from (4) with $\alpha=\theta$ we get

$$
H\left(x_{0}\right)=H(x)+O\left(X^{2 \theta-1+\varepsilon}\right),
$$

hence from (6) with $\alpha=\theta$ we obtain

$$
|\Delta(x, H)| \geq \delta H(x)-\frac{9}{2 \theta} c H(X)+O\left(X^{2 \theta-1+\varepsilon}\right) \geq \delta H(x)-\frac{5}{\theta} c H(X) \geq \delta^{\prime} H(x)
$$

by choosing $c=\left(\delta-\delta^{\prime}\right) \theta / 5$, since $H(x)$ is increasing. Hence $x \in E_{\delta^{\prime}}(X, H)$ and the lemma follows.

Lemma 1 is part (i) of Theorem 1 of Bazzanella and Perelli, see [2], and essentially says that if we have a single exception in $E_{\delta}(X, H)$, with a fixed $\delta$, then we necessarily have an interval of exceptions in $E_{\delta^{\prime}}(X, H)$, with $\delta^{\prime}$ a little smaller than $\delta$.

We now present the necessary results about the conditional and unconditional bounds for the exceptional set for the number of primes in short intervals. With this in mind, we consider $H(x)$ of type $\theta$ and define the functions

$$
\mu_{\delta}(\theta)=\inf \left\{\xi \geq 0:\left|E_{\delta}(X, H)\right| \ll_{\delta} X^{\xi}\right\}
$$

and

$$
\mu(\theta)=\sup _{\delta>0} \mu_{\delta}(\theta)
$$

Our results are as follows.

LEMMA 2. There exists a constant $\eta>0$ such that

$$
\mu(\theta) \leq \frac{(11-6 \theta)}{10} \text { if } \frac{1}{6}<\theta \leq \frac{1}{6}+\eta .
$$


PROOF. In order to prove Lemma 2 we use the classical explicit formula (see Davenport [3, Ch. 17]) to write

$$
\psi(x+H(x))-\psi(x)-H(x)=-\sum_{|\gamma| \leq T} x^{\rho} c_{\rho}(x)+O\left(\frac{X \log ^{2} X}{T}\right),
$$

uniformly for all $X \leq x \leq 2 X$, where $10 \leq T \leq X, \rho=\beta+i \gamma$ runs over the nontrivial zeros of $\zeta(s)$,

$$
c_{\rho}(x)=\frac{(1+H(x) / x)^{\rho}-1}{\rho} \text { and } c_{\rho}(x) \ll \min \left(\frac{H(X)}{X}, \frac{1}{|\gamma|}\right) .
$$

Let $H(x)$ be of type $\theta$. Choose

$$
T=\frac{X}{H(X)} \log ^{3} X
$$

and use the theorem of Montgomery (see Theorem 11.3 of Ivic [9]) which asserts that

$$
N(\sigma, T) \ll T^{1600(1-\sigma)^{3 / 2}} \log ^{15} T
$$

for every $152 / 155 \leq \sigma \leq 1$. From (9)-(11) and Vinogradov's zero-free region (see Titchmarsh [12, Ch. 6]) we deduce by a standard argument that there exists a constant $d>0$ such that

$$
\sum_{\substack{|\gamma| \leq T \\ \beta \notin I}} x^{\rho} c_{\rho}(x) \ll \frac{H(X)}{X} \log X \max _{\sigma \notin I} X^{\sigma} N(\sigma, T) \ll \frac{H(X)}{\log X},
$$

where $I=[1 / 2,1-d]$, uniformly for all $X \leq x \leq 2 X$.

Again by a standard argument, from (9), (10) and the Ingham-Huxley density estimates which assert that for every $\varepsilon>0$ we have

$$
N(\sigma, T) \ll \begin{cases}T^{3(1-\sigma) /(2-\sigma)+\varepsilon}, & \frac{1}{2} \leq \sigma \leq \frac{3}{4}, \\ T^{3(1-\sigma) /(3 \sigma-1)+\varepsilon}, & \frac{3}{4} \leq \sigma \leq 1\end{cases}
$$

we obtain

$$
\int_{X}^{2 X}\left|\sum_{\substack{|\gamma| \leq T \\ \beta \in I}} x^{\rho} c_{\rho}(x)\right|^{2} d x \ll X^{2 \theta-1+\varepsilon} \max _{\sigma \in I} X^{2 \sigma} N(\sigma, T) \ll X^{(11+14 \theta) / 10+\varepsilon},
$$

for sufficiently small $\eta>0$ and $1 / 6<\theta \leq 1 / 6+\eta$. Hence, for every $\varepsilon>0$ and $\delta>0$,

$$
\left|E_{\delta}(X, H)\right| \ll X^{(11-6 \theta) / 10+\varepsilon},
$$

and so the lemma is proved. 
We observe that we can take $d=2.5 \cdot 10^{-7}$ and then $\eta=3.125 \cdot 10^{-7}$. The value of $\eta$ could be somewhat increased by using an optimized version of density estimate (11).

Lemma 3. Assume (2). Then

$$
\mu(\theta) \leq \frac{7}{5}(1-\theta) \quad \text { if } \frac{23}{48}<\theta<\frac{7}{12} .
$$

ProOF. Let $H(x)$ be of type $\theta$ and

$$
T=\frac{X}{H(X)} \log ^{3} X
$$

Following the method of Heath-Brown [5], we can write

$$
\int_{X}^{2 X}|\psi(x+H(x))-\psi(x)-H(x)+\Sigma|^{4} d x \ll X^{4 \theta-3+\varepsilon} \max _{1 / 2 \leq \sigma \leq 1} X^{4 \sigma} N^{*}(\sigma, T),
$$

with $\Sigma=o(H(X))$. Assuming (2) and using the Ingham-Huxley zero density estimates, the above estimate implies that

$$
\begin{aligned}
\left|E_{\delta}(X, H)\right| \ll & X^{-3+\varepsilon} \max _{1 / 2 \leq \sigma \leq 1} X^{4 \sigma} N^{*}(\sigma, T) \ll X^{-3+\varepsilon} \max _{1 / 2 \leq \sigma \leq 1} X^{4 \sigma} \frac{N(\sigma, T)^{4}}{T} \\
\ll & X^{\theta-4+\varepsilon}\left(\max _{1 / 2 \leq \sigma \leq 3 / 4} X^{4 \sigma} T^{12(1-\sigma) /(2-\sigma)}\right. \\
& \left.+\max _{3 / 4 \leq \sigma \leq 1} X^{4 \sigma} T^{12(1-\sigma) /(3 \sigma-1)}\right),
\end{aligned}
$$

for every $\delta>0$ and $\varepsilon>0$. With $23 / 48<\theta<7 / 12$ the maximum is attained at $\sigma=3 / 4$, so

$$
\left|E_{\delta}(X, H)\right| \ll X^{\frac{7}{5}(1-\theta)+\varepsilon},
$$

for every $\delta>0$ and $\varepsilon>0$. This completes the proof of the lemma.

Lemma 4. Assume the Lindelöf hypothesis, let $\varepsilon>0$ and $\delta>0$. For every $H \geq 1$,

$$
\left|E_{\delta}(X, H)\right| \ll \frac{X^{1+\varepsilon}}{H(X)} .
$$

Lemma 4 may be proved along the same lines as Yu [13, Lemma B].

To deal with the problem of estimating the exceptional set for the distribution of primes in intervals $\left[n^{\alpha}, n^{\alpha}+H\right] \subset[N, 2 N]$, suppose that $H(x)$ is of type $\theta$, let

$$
\Delta(n, H, \alpha)=\psi\left(n^{\alpha}+H\left(n^{\alpha}\right)\right)-\psi\left(n^{\alpha}\right)-H\left(n^{\alpha}\right),
$$

and define the set

$$
A_{\delta}(N, H, \alpha)=\left\{N^{1 / \alpha} \leq n \leq(2 N)^{1 / \alpha}:|\Delta(n, H, \alpha)| \geq \delta H\left(n^{\alpha}\right)\right\},
$$


that contains the exceptions, if any, to the expected asymptotic formula for the number of primes in intervals of type $\left[n^{\alpha}, n^{\alpha}+H\left(n^{\alpha}\right)\right] \subset[N, 2 N]$. Our last lemmas allow us to link $\left|A_{\delta}(N, H, \alpha)\right|$ to the exceptional set for the distribution of primes in short intervals.

LEMMA 5. Let $H(x)$ be of type $\theta$, with $1 / 6<\theta<7 / 12$. Then for every $\delta>0$ we have the following results:

(i)

$$
\left|A_{\delta}(N, H, \alpha)\right|=o\left(N^{1 / \alpha}\right) \quad \text { if } 1<\alpha \leq \frac{6}{5} .
$$

(ii)

$$
\left|A_{\delta}(N, H, \alpha)\right| \ll \frac{\left|E_{\delta / 2}(N, H)\right| f(N) \log ^{2} N}{H(N)}+o\left(N^{1 / \alpha}\right) \quad \text { if } \alpha>\frac{6}{5},
$$

with $f(N) \rightarrow \infty$ arbitrarily slowly.

PROOF. Recalling the explicit formula for $\psi(x)$ and putting

$$
T=\frac{N}{H(N)} f(N) \log ^{2} N
$$

where $f(N) \rightarrow \infty$ arbitrarily slowly, we have

$$
\begin{aligned}
\psi\left(n^{\alpha}+H\left(n^{\alpha}\right)\right)-\psi\left(n^{\alpha}\right)-H\left(n^{\alpha}\right) & =-\sum_{|\gamma|<T} n^{\alpha \rho} c_{\rho}(n)+o(H(N)) \\
& =-\sum_{\substack{|\gamma|<T \\
\beta \in I}} n^{\alpha \rho} c_{\rho}(n)+o(H(N)),
\end{aligned}
$$

where $d$ and $I=[1 / 2,1-d]$ are defined as in the proof of Lemma 2,

$$
c_{\rho}(n)=\frac{1-\left(1+H\left(n^{\alpha}\right) n^{-\alpha}\right)^{\rho}}{\rho} \quad \text { and } \quad c_{\rho}(n) \ll \min \left(\frac{H(N)}{N}, \frac{1}{|\gamma|}\right) \text {. }
$$

Further we divide the interval $I$ into $O(\log N)$ subintervals $I_{j}$ of the form

$$
I_{j}=\left[\frac{j-1}{\log N}, \frac{j}{\log N}\right] \cap I
$$

On applying Cauchy's inequality we find

$$
\left|\sum_{\substack{|\gamma|<T \\ \beta \in I}} n^{\alpha \rho} c_{\rho}(n)\right|^{2} \ll \log N \sum_{j}\left|\sum_{\substack{|\gamma|<T \\ \beta \in I_{j}}} n^{\alpha \rho} c_{\rho}(n)\right|^{2},
$$


and so we get

$$
\begin{aligned}
H(N)^{2}\left|A_{\delta}(N, H, \alpha)\right| & \ll \sum_{n \in A_{\delta}(N, H, \alpha)}\left|\psi\left(n^{\alpha}+H\left(n^{\alpha}\right)\right)-\psi\left(n^{\alpha}\right)-H\left(n^{\alpha}\right)+o(H(N))\right|^{2} \\
& \leq \sum_{N^{1 / \alpha} \leq n \leq(2 N)^{1 / \alpha}}\left|\sum_{\substack{|\gamma|<T \\
\beta \in I}} n^{\alpha \rho} c_{\rho}(n)\right|^{2} \\
& \ll \log N \sum_{N^{1 / \alpha} \leq n \leq(2 N)^{1 / \alpha}} \sum_{j}\left|\sum_{\substack{|\gamma|<T \\
\beta \in I_{j}}} n^{\alpha \rho} c_{\rho}(n)\right|^{2} .
\end{aligned}
$$

Squaring and using partial summation we then have

$$
\begin{aligned}
\left|A_{\delta}(N, H, \alpha)\right| & \ll \frac{\log N}{H(N)^{2}} \sum_{N^{1 / \alpha} \leq n \leq(2 N)^{1 / \alpha}} \sum_{j} \sum_{\substack{|\gamma|<T \\
\beta \in I_{j}}} \sum_{\substack{\left|\gamma^{\prime}\right|<T \\
\beta^{\prime} \in I_{j}}} n^{\alpha\left(\rho+\overline{\rho^{\prime}}\right)} c_{\rho}(n) \overline{c_{\rho^{\prime}}(n)} \\
& \ll \frac{\log N}{N^{2}} \sum_{j} N^{2 j / \log N} \sum_{\substack{|\gamma|<T \\
\beta \in I_{j}}} \sum_{\substack{\left|\gamma^{\prime}\right|<T \\
\beta^{\prime} \in I_{j}}}|S|
\end{aligned}
$$

where

$$
\begin{gathered}
S=\sum_{N^{1 / \alpha} \leq n \leq\left(N_{1}\right)^{1 / \alpha} n^{\alpha i\left(\gamma-\gamma^{\prime}\right)}}=\sum_{N^{1 / \alpha} \leq n \leq\left(N_{1}\right)^{1 / \alpha}} \mathrm{e}(g(n)), \\
\mathrm{e}(x)=\mathrm{e}^{2 \pi i x}, \quad g(x)=\frac{\alpha\left(\gamma-\gamma^{\prime}\right)}{2 \pi} \log x
\end{gathered}
$$

and $N \leq N_{1} \leq 2 N$.

Let

$$
H(N) \geq \frac{2 \alpha}{\pi} N^{1-1 / \alpha} f(N) \log ^{2} N,
$$

with $f(N) \rightarrow \infty$ arbitrarily slowly. Using the theorem of Kusmin-Landau (see Graham and Kolesnik [4, Theorem 2.1]) and the trivial bound, one finds that

$$
|S| \ll \frac{N^{1 / \alpha}}{\left|\gamma-\gamma^{\prime}\right|} \quad \text { and } \quad|S| \ll N^{1 / \alpha},
$$

and hence

$$
\begin{aligned}
\left|A_{\delta}(N, H, \alpha)\right| \ll & \frac{\log N}{N^{2}} \sum_{j} N^{2 j / \log N} \sum_{\substack{|\gamma|<T \\
\beta \in I_{j}}} \sum_{\substack{\left|\gamma^{\prime}\right|<T \\
\beta^{\prime} \in I_{j},\left|\gamma-\gamma^{\prime}\right| \leq 1}} N^{1 / \alpha} \\
& +\frac{\log N}{N^{2}} \sum_{j} N^{2 j / \log N} \sum_{\substack{|\gamma|<T \\
\beta \in I_{j}}} \sum_{\substack{\left|\gamma^{\prime}\right|<T \\
\beta^{\prime} \in I_{j},\left|\gamma-\gamma^{\prime}\right|>1}} \frac{N^{1 / \alpha}}{\left|\gamma-\gamma^{\prime}\right|},
\end{aligned}
$$


which implies

$$
\left|A_{\delta}(N, H, \alpha)\right| \ll \frac{N^{1 / \alpha}}{N^{2}} \log ^{3} N\left(\sum_{j} \sum_{\substack{|\gamma|<T \\ \beta \in I_{j}}} N^{2 j / \log N}\right) .
$$

For every $1<\alpha \leq 6 / 5$ and $H(x)$ of type $\theta$ with $1 / 6<\theta<7 / 12$, and for every $\alpha>6 / 5$ and $H(x)$ satisfying (14), it follows by a standard argument and the InghamHuxley zero density estimates that

$$
\sum_{j} \sum_{\substack{|\gamma|<T \\ \beta \in I_{j}}} N^{2 j / \log N} \ll \max _{\sigma \in I} N^{2 \sigma} N(\sigma, T) \ll \frac{N^{2}}{\log ^{A} N},
$$

for every $A>0$. From (15) and (16), it follows that

$$
\left|A_{\delta}(N, H, \alpha)\right|=o\left(N^{1 / \alpha}\right)
$$

for every $1<\alpha \leq 6 / 5$ and for every $\alpha>6 / 5$ with

$$
H(N) \geq \frac{2 \alpha}{\pi} N^{1-1 / \alpha} f(N) \log ^{2} N .
$$

Finally, let $\alpha>6 / 5$ and

$$
H(N)<\frac{2 \alpha}{\pi} N^{1-1 / \alpha} f(N) \log ^{2} N .
$$

To deal with this small $H$ we observe that if $n \in A_{\delta}(N, H, \alpha)$ then $N \leq n^{\alpha} \leq 2 N$ and

$$
\left|\psi\left(n^{\alpha}+H\left(n^{\alpha}\right)\right)-\psi\left(n^{\alpha}\right)-H\left(n^{\alpha}\right)\right| \geq \delta H\left(n^{\alpha}\right) .
$$

Thus $n^{\alpha} \in E_{\delta}(N, H)$. By Lemma 1 we find a constant $c>0$ such that

$$
\left[n^{\alpha}-c H(N), n^{\alpha}+c H(N)\right] \cap[N, 2 N] \subset E_{\delta / 2}(N, H) .
$$

We now consider $m \in A_{\delta}(N, H, \alpha)$, with $|m-n| \geq(2 / \pi) f(N) \log ^{2} N$ and similarly we get $m^{\alpha} \in E_{\delta}(N, H)$ and then

$$
\left[m^{\alpha}-c H(N), m^{\alpha}+c H(N)\right] \cap[N, 2 N] \subset E_{\delta / 2}(N, H),
$$

again by Lemma 1 . Since

$$
\left|m^{\alpha}-n^{\alpha}\right| \geq|m-n| \alpha N^{1-1 / \alpha} \geq \frac{2 \alpha}{\pi} N^{1-1 / \alpha} f(N) \log ^{2} N>H(N),
$$

we may deduce that

$$
\left[m^{\alpha}-c H(N), m^{\alpha}+c H(N)\right] \cap\left[n^{\alpha}-c H(N), n^{\alpha}+c H(N)\right]=\emptyset,
$$

for $c$ suitable small. This leads to the bound

$$
\left|A_{\delta}(N, H, \alpha)\right| \ll \frac{\left|E_{\delta / 2}(N, H)\right| f(N) \log ^{2} N}{H(N)},
$$

for every $\delta>0$, which proves the lemma. 
LEMMA 6. Assume the Lindelöf hypothesis. Let $H(x)$ be of type $\theta$, with $0<\theta<1 / 2$. Then, for every $\delta>0$ and $\alpha>1$,

$$
\left|A_{\delta}(N, H, \alpha)\right| \ll \frac{\left|E_{\delta / 2}(N, H)\right| f(N) \log ^{2} N}{H(N)}+o\left(N^{1 / \alpha}\right)
$$

with $f(N) \rightarrow \infty$ arbitrarily slowly.

PROOF. We follow the proof of the Lemma 5 until the equation (15). Under the assumption of the Lindelöf hypothesis, which states that the Riemann zeta function satisfies

$$
\zeta(\sigma+i t) \ll t^{\eta} \quad\left(\sigma \geq \frac{1}{2}, t \geq 2\right)
$$

for any $\eta>0$, we have

$$
N(\sigma, T) \ll \begin{cases}T^{(2+4 \eta)(1-\sigma)}(\log T)^{M}, & 0 \leq \sigma \leq 1, \\ T^{3 \eta(1-\sigma) /(\sigma-3 / 4)}(\log T)^{M}, & \frac{3}{4}<\sigma \leq 1,\end{cases}
$$

with $T \geq 2$ and $M$ suitable absolute constant (see Lemma 3 of Yu [13]). From (17) it follows that the bound (16) holds for every

$$
H(N) \geq \frac{2 \alpha}{\pi} N^{1-1 / \alpha} f(N) \log ^{2} N
$$

and $\alpha>1$. We can conclude the proof by dealing with smaller values of $H$ in the same way as in the proof of Lemma 5.

\section{Proof of the Theorem 1}

By the case (i) of Lemma 5, we can take

$$
c(\alpha)=\frac{1}{6} \quad \text { if } 1<\alpha \leq \frac{6}{5} .
$$

For all $\alpha>6 / 5$, by (ii) of Lemma 5 ,

$$
\left|A_{\delta}(N, H, \alpha)\right| \ll \frac{\left|E_{\delta / 2}(N, H)\right| f(N) \log ^{2} N}{H(N)}+o\left(N^{1 / \alpha}\right),
$$

for every $H(x)$ of type $\theta$, with $1 / 6<\theta<7 / 12$. Furthermore, by Lemma 2 there exists $\eta>0$ such that

$$
\left|E_{\delta / 2}(N, H)\right| \ll N^{(11-6 \theta) / 10+\varepsilon},
$$

for every

$$
\frac{1}{6}<\theta \leq \frac{1}{6}+\eta
$$

and every $H(x)$ of type $\theta$. These estimates together yield

$$
\left|A_{\delta}(N, H, \alpha)\right| \ll N^{(11-16 \theta) / 10+\varepsilon}+o\left(N^{1 / \alpha}\right),
$$


and

$$
\left|A_{\delta}(N, H, \alpha)\right|=o\left(N^{1 / \alpha}\right),
$$

for every

and sufficiently small $\alpha>6 / 5$.

$$
\theta>\frac{11 \alpha-10}{16 \alpha}
$$

It follows that

$$
c(\alpha)=\frac{11 \alpha-10}{16 \alpha} \text { if } \frac{6}{5}<\alpha \leq \frac{6}{5}+\Delta,
$$

for suitable positive constant $\Delta$. From the explicit value for $\eta$ available from the Lemma 2, we can state that an admissible value is $\Delta=7.2 \cdot 10^{-7}$.

To estimate $c(\alpha)$ for large values of $\alpha$ we need to follow a quite different method. In a similar way as in the proof of Lemma 5 , we let

$$
T=\frac{N}{H(N)} \log ^{3} N
$$

and write

$$
\psi\left(n^{\alpha}+H\left(n^{\alpha}\right)\right)-\psi\left(n^{\alpha}\right)-H\left(n^{\alpha}\right)=-\sum_{\substack{|\gamma|<T \\ \beta \in I}} n^{\alpha \rho} c_{\rho}(n)+o(H(N)),
$$

where $I=[1 / 2,1-d]$, for a suitable positive constant $d$. Next we divide the interval $I$ into $O(\log N)$ subintervals $I_{j}$ of the form

$$
I_{j}=\left[\frac{j-1}{\log N}, \frac{j}{\log N}\right] \cap I .
$$

Using Hölder's inequality, we get

$$
\left|\sum_{\substack{|\gamma|<T \\ \beta \in I}} n^{\alpha \rho} c_{\rho}(n)\right|^{4} \ll \log ^{3} N \sum_{j}\left|\sum_{\substack{|\gamma|<T \\ \beta \in I_{j}}} n^{\alpha \rho} c_{\rho}(n)\right|^{4}
$$

and then we can deduce

$$
\begin{aligned}
& \left|A_{\delta}(N, H, \alpha)\right| \ll \frac{\log ^{3} N}{H(N)^{4}} \sum_{N^{1 / \alpha} \leq n \leq(2 N)^{1 / \alpha}} \sum_{j}\left|\sum_{\substack{|\gamma|<T \\
\beta \in I_{j}}} n^{\alpha \rho} c_{\rho}(n)\right|^{4} \\
& \quad \ll \frac{\log ^{3} N}{H(N)^{4}} \sum_{N^{1 / \alpha} \leq n \leq(2 N)^{1 / \alpha}} \sum_{j} \sum_{\substack{|\gamma|<T \\
\beta \in I_{j}}} \sum_{\substack{\left|\gamma^{\prime}\right|<T \\
\beta^{\prime} \in I_{j}}} \sum_{\substack{\left|\gamma^{\prime \prime}\right|<T \\
\beta^{\prime \prime} \in I_{j}}} n_{\substack{\left|\gamma^{\prime \prime \prime}\right|<T \\
\beta^{\prime \prime \prime} \in I_{j}}} n^{\alpha\left(\rho+\rho^{\prime}+\overline{\rho^{\prime \prime}}+\overline{\rho^{\prime \prime \prime}}\right)} C_{n} \\
& \quad \ll \frac{\log ^{3} N}{N^{4}} \sum_{j} N^{4 j / \log N} \sum_{\substack{|\gamma|<T \\
\beta \in I_{j}}} \sum_{\substack{\left|\gamma^{\prime}\right|<T \\
\beta^{\prime} \in I_{j}}} \sum_{\substack{\left|\gamma^{\prime \prime}\right|<T \\
\beta^{\prime \prime} \in I_{j}}} \sum_{\substack{\left|\gamma^{\prime \prime \prime}\right|<T \\
\beta^{\prime \prime \prime} \in I_{j}}}|S| \\
& =V_{1}+V_{2},
\end{aligned}
$$


where

$$
\begin{gathered}
C_{n}=c_{\rho}(n) c_{\rho^{\prime}}(n) \overline{c_{\rho^{\prime \prime}}(n) c_{\rho^{\prime \prime \prime}}(n),}, \\
S=\sum_{N^{1 / \alpha} \leq n \leq\left(N_{1}\right)^{1 / \alpha}} \mathrm{e}(g(n)), \quad g(x)=\frac{\alpha\left(\gamma+\gamma^{\prime}-\gamma^{\prime \prime}-\gamma^{\prime \prime \prime}\right)}{2 \pi} \log x, \\
V_{1}=\frac{\log ^{3} N}{N^{4}} \sum_{j} N^{4 j / \log N} \sum_{\substack{|\gamma|<T \\
\beta \in I_{j}}} \sum_{\substack{\left|\gamma^{\prime}\right|<T \\
\beta^{\prime} \in I_{j}}} \sum_{\substack{\left|\gamma^{\prime \prime}\right|<T \\
\beta^{\prime \prime} \in I_{j} \\
\left|\gamma^{\prime \prime \prime}\right|<T \\
\beta^{\prime \prime \prime} \in I_{j} \\
\left|\gamma+\gamma^{\prime}-\gamma^{\prime \prime}-\gamma^{\prime \prime \prime}\right| \leq(\pi / \alpha) N^{1 / \alpha}}}
\end{gathered}
$$

and

$$
V_{2}=\frac{\log ^{3} N}{N^{4}} \sum_{j} N^{4 j / \log N} \sum_{\substack{|\gamma|<T \\ \beta \in I_{j}}} \sum_{\substack{\left|\gamma^{\prime}\right|<T \\ \beta^{\prime} \in I_{j}}} \sum_{\substack{\left|\gamma^{\prime \prime}\right|<T \\ \beta^{\prime \prime} \in I_{j}}} \sum_{\substack{\left|\gamma^{\prime \prime \prime}\right|<T \\ \beta^{\prime \prime \prime} \in I_{j} \\\left|\gamma+\gamma^{\prime}-\gamma^{\prime \prime}-\gamma^{\prime \prime \prime}\right|>(\pi / \alpha) N^{1 / \alpha}}}
$$

We first proceed to estimate $V_{1}$. For the terms in the inner sum with

$$
\left|\gamma+\gamma^{\prime}-\gamma^{\prime \prime}-\gamma^{\prime \prime \prime}\right|<1
$$

we can estimate $|S|$ using the trivial bound. For the terms with

$$
1 \leq\left|\gamma+\gamma^{\prime}-\gamma^{\prime \prime}-\gamma^{\prime \prime \prime}\right| \leq \frac{\pi}{\alpha} N^{1 / \alpha}
$$

we can use the Kusmin-Landau theorem. Hence we obtain the estimate

$$
S \ll \frac{N^{1 / \alpha}}{1+\left|\gamma+\gamma^{\prime}-\gamma^{\prime \prime}-\gamma^{\prime \prime \prime}\right|},
$$

which, by Heath-Brown's method [5], implies

$$
V_{1} \ll \frac{N^{1 / \alpha} \log ^{5} N}{N^{4}} \max _{\sigma \in I} N^{4 \sigma} N^{*}(\sigma, T) .
$$

For $H(x)$ of type $\theta$, with $0.342<\theta<7 / 12$, Heath-Brown's zero-density estimates

$$
N^{*}(\sigma, T) \ll \begin{cases}T^{(10-11 \sigma) /(2-\sigma)+\varepsilon}, & \frac{1}{2} \leq \sigma \leq \frac{2}{3}, \\ T^{(18-19 \sigma) /(4-2 \sigma)+\varepsilon}, & \frac{2}{3} \leq \sigma \leq \frac{3}{4}, \\ T^{12(1-\sigma) /(4 \sigma-1)+\varepsilon}, & \frac{3}{4} \leq \sigma \leq 1,\end{cases}
$$

[6, Theorem 2] give upper bounds for $N^{4 \sigma} N^{*}(\sigma, T)$ that attain their maximum at $\sigma=1-d$. A short calculation then shows that

$$
\max _{\sigma \in I} N^{4 \sigma} N^{*}(\sigma, T) \ll \frac{N^{4}}{(\log N)^{A}},
$$


for every $A>0$. Hence we conclude that

$$
V_{1}=o\left(N^{1 / \alpha}\right)
$$

for every $0.342<\theta<7 / 12$.

Now we turn to estimating $V_{2}$. Let $(k, l)$ be an exponent pair, then

$$
S \ll\left(\frac{\left|\gamma+\gamma^{\prime}-\gamma^{\prime \prime}-\gamma^{\prime \prime \prime}\right|}{N^{1 / \alpha}}\right)^{k}\left(N^{1 / \alpha}\right)^{l} \ll\left(\frac{T}{N^{1 / \alpha}}\right)^{k} N^{l / \alpha} \ll N^{(k \alpha(1-\theta)-k+l) / \alpha+\varepsilon},
$$

for every $\varepsilon>0$ and $H(x)$ of type $\theta$. This yields

$$
\begin{aligned}
V_{2} & \ll N^{(k \alpha(1-\theta)-k+l) / \alpha-4+\varepsilon} \sum_{j}\left(\sum_{\substack{|\gamma|<T \\
\beta \in I_{j}}} N^{j / \log N}\right)^{4} \\
& \ll N^{(k \alpha(1-\theta)-k+l) / \alpha-4+\varepsilon}\left(\max _{\sigma} N^{\sigma} N(\sigma, T)\right)^{4} .
\end{aligned}
$$

For $H$ of type $\theta$, with

$$
\frac{23}{48}<\theta<\frac{7}{12},
$$

the density estimates of Ingham-Huxley give upper bounds for $N^{\sigma} N(\sigma, T)$ that attain their maximum at $\sigma=3 / 4$. So we may deduce

$$
V_{2} \ll N^{(k \alpha(1-\theta)-k+l) / \alpha-4+\varepsilon} N^{3+12(1-\theta) / 5} .
$$

The above bound is $o\left(N^{1 / \alpha}\right)$ for every

$$
\theta>1-\frac{5(1+\alpha-l+k)}{\alpha(5 k+12)}
$$

if $(k, l)$ is an exponent pair, $H$ is of type $\theta$ and $\alpha$ is sufficiently large. Thus we can select

$$
c(\alpha)=1-\sup _{(k, l)} \frac{5(1+\alpha-l+k)}{\alpha(5 k+12)}
$$

where $(k, l)$ runs over the exponent pairs. Since all exponent pairs $(k, l)$ have $0 \leq k \leq 1 / 2 \leq l$, we obtain

$$
1-\frac{5}{12} \frac{1+\alpha}{\alpha}<1-\sup _{(k, l)} \frac{5(1+\alpha-l+k)}{\alpha(5 k+12)}=c(\alpha),
$$

which implies (19) if $\alpha \geq 4$. On the other hand from the exponent pairs

$$
A^{i-1} B(0,1)=\left(\frac{1}{2\left(2^{i}-1\right)}, 1-\frac{i}{2\left(2^{i}-1\right)}\right)
$$


where

$$
i=\left[\frac{5 \alpha}{12}\right]
$$

we get

$$
1-\sup _{(k, l)} \frac{5(1+\alpha-l+k)}{\alpha(5 k+12)}=c(\alpha)<\frac{7}{12}
$$

and then, as one might expect, we conclude that

$$
\lim _{\alpha \rightarrow+\infty} c(\alpha)=\frac{7}{12}
$$

This completes the proof of Theorem 1 .

Note. We are able to obtain the function $c(\alpha)$, in a suitable interval of $\alpha$, from every estimate of the counting function $N(\sigma, T)$ in a fixed interval of $\sigma$. As an example, if we recall that

$$
N(\sigma, T) \ll T^{9(1-\sigma) /(7 \sigma-1)} \log ^{C} T,
$$

with $41 / 53 \leq \sigma \leq 1$ and $C$ suitable constant (see Theorem 11.4 of Ivić [9]), we can choose $H$ of type $\theta, d=(9 \theta-3) / 7-\xi$ with $\xi>0$, in (12) of Lemma 2, using the Ingham-Huxley density estimates and (21) we can obtain an estimate of $\left|E_{\delta}(X, H)\right|$. Hence, from Lemma 5, we can obtain

$$
c(\alpha)= \begin{cases}\frac{5}{8}-\frac{7}{16 \alpha} & \text { if } \frac{3}{2}<\alpha \leq \frac{3339}{1138} \\ \frac{1969}{2809}-\frac{35}{53 \alpha} & \text { if } \frac{3339}{1138} \leq \alpha \leq 3.447\end{cases}
$$

that cover a great part of the gap between $6 / 5+\Delta$ and 4 . Along the same lines we can obtain a large number of possible function $c(\alpha)$, for every $\alpha>1$.

\section{Proof of Theorems 2, 3 and 4}

In order to prove Theorem 2 we assume (2) and use Lemma 5 to see that

$$
\left|A_{\delta}(N, H, \alpha)\right| \ll \frac{\left|E_{\delta / 2}(N, H)\right| f(N) \log ^{2} N}{H(N)}+o\left(N^{1 / \alpha}\right),
$$

for every $H(x)$ of type $\theta$, with $1 / 6<\theta<7 / 12$. So by Lemma 3

$$
\left|E_{\delta / 2}(N, H)\right| \ll N^{\frac{7}{5}(1-\theta)+\varepsilon},
$$

with $H(x)$ of type $\theta$ and $23 / 48<\theta<7 / 12$. The last two estimates together yield

$$
\left|A_{\delta}(N, H, \alpha)\right| \ll N^{\frac{7}{5}-\frac{12}{5} \theta+\varepsilon}+o\left(N^{1 / \alpha}\right),
$$


so that

$$
\left|A_{\delta}(N, H, \alpha)\right|=o\left(N^{1 / \alpha}\right),
$$

for every

$$
\theta>\frac{7}{12}-\frac{5}{12 \alpha} \text { and } \quad \frac{23}{48}<\theta<\frac{7}{12}
$$

Then we can define

$$
c(\alpha)=\frac{7}{12}-\frac{5}{12 \alpha} \quad \text { if } \alpha \geq 4
$$

This completes the proof of Theorem 2 .

Similarly we can prove Theorem 3, using Lemmas 6 and 4 instead of Lemmas 5 and 3 , so obtaining

$$
\left|A_{\delta}(N, H, \alpha)\right| \ll N^{1-2 \theta+\varepsilon}+o\left(N^{1 / \alpha}\right),
$$

so that

$$
\left|A_{\delta}(N, H, \alpha)\right|=o\left(N^{1 / \alpha}\right)
$$

for every

$$
\theta>\frac{1}{2}\left(1-\frac{1}{\alpha}\right)
$$

Then we can choose

$$
c(\alpha)=\frac{1}{2}\left(1-\frac{1}{\alpha}\right) \quad \text { if } \alpha>1 .
$$

This completes the proof of Theorem 3 .

To prove Theorem 4 we recall that Selberg [11] proved, under the assumption of the Riemann hypothesis, that

$$
\int_{X}^{2 X}|\psi(x+H)-\psi(x)-H|^{2} d x \ll H X \log ^{2} X,
$$

for all $H \geq 10$, which implies

$$
\left|E_{\delta}(N, H)\right| \ll \frac{N}{H(N)} \log ^{2} N,
$$

for every $\delta>0$. In conjunction with Lemma 6 , this gives

$$
\left|A_{\delta}(N, H, \alpha)\right| \ll \frac{N \log ^{4} N}{H(N)^{2}} f(N)+o\left(N^{1 / \alpha}\right),
$$

with $f(N) \rightarrow \infty$ arbitrarily slowly, so that

$$
\left|A_{\delta}(N, H, \alpha)\right|=o\left(N^{1 / \alpha}\right),
$$

with

$$
H(N)>N^{\frac{1}{2}\left(1-\frac{1}{\alpha}\right)} f(N) \log ^{2} N,
$$

for every $\alpha>1$ and $\delta>0$. This completes the proof of Theorem 4 . 


\section{Acknowledgement}

We are particularly indebted to the referee for a very thorough reading and some helpful suggestions.

\section{References}

[1] D. Bazzanella, 'Primes between consecutive square', Arch. Math. 75 (2000), 29-34.

[2] D. Bazzanella and A. Perelli, 'The exceptional set for the number of primes in short intervals', J. Number Theory 80 (2000), 109-124.

[3] H. Davenport, Multiplicative Number Theory, 2nd edn, Graduate Texts in Mathematics, 74 (Springer, Berlin, 1980).

[4] S. W. Graham and G. Kolesnik, Van der Corput's Method of Exponential Sums (Cambridge University Press, Cambridge, 1991).

[5] D. R. Heath-Brown, 'The differences between consecutive primes II', J. London Math. Soc. (2) 19 (1979), 207-220.

[6] _ 'Zero density estimates for the Riemann zeta-function and Dirichlet $L$-function', J. London Math. Soc. (2) 19 (1979), 221-232.

[7] _ - 'The number of primes in a short interval', J. Reine Angew. Math. 389 (1988), 22-63.

[8] M. N. Huxley, 'On the difference between consecutive primes', Invent. Math. 15 (1972), 164-170.

[9] A. Ivić, The Riemann Zeta-Function (John Wiley and Sons, New York, 1985).

[10] H. L. Montgomery and R .C. Vaughan, 'The large sieve', Mathematika 20 (1973), 119-134.

[11] A. Selberg, 'On the normal density of primes in small intervals, and the difference between consecutive primes', Arc. Math. Naturvid. 47(6) (1943), 87-105.

[12] E. C. Titchmarsh, The Theory of the Riemann Zeta-function, 2nd edn (Oxford University Press, Oxford, 1986).

[13] G. Yu, 'The differences between consecutive primes', Bull. London Math. Soc. 28(3) (1996), $242-248$.

[14] A. Zaccagnini, 'Primes in almost all short intervals', Acta Arith. 84 (1998), 225-244.

DANILO BAZZANELLA, Dipartimento di Matematica, Politecnico di Torino, 10129 Torino, Italy

e-mail: danilo.bazzanella@polito.it 